\title{
APLICAÇÃO DE CHECKLIST SOBRE CUIDADOS INTRAPARTO NO PARTO NORMAL
}

\section{APPLICATION OF CHECKLIST ON INTRAPARTUM CARE IN NORMAL CHILDREN}

\author{
Luana Sousa de Carvalho ${ }^{1} *$ Beatriz Davini Sales Rebouças ${ }^{2} *$ Luana Silva de Sousa $^{3}$ \\ Antonio Rodrigues Ferreira Junior ${ }^{4} *$ Rhanna Emanuela Fontenele Lima de Carvalho ${ }^{5}$
}

\begin{abstract}
RESUMO
Diante do contexto de uso das tecnologias para auxiliar as boas práticas no parto, objetivou-se por meio desse estudo, aplicar o checklist de cuidados intraparto no parto vaginal. Estudo descritivo, transversal de abordagem quantitativa, desenvolvido durante os meses de novembro e dezembro de 2020, nas salas de parto de dois hospitais, um de nível secundário e outro terciário. A amostra do estudo foi constituída por conveniência totalizando um quantitativo de 40 profissionais. Os dados do instrumento foram agrupados, tabulados e transferidos para o programa Excel. A análise descritiva dos dados foi realizada por meio de frequências relativas e absolutas. Observou-se que os profissionais seguiram as recomendações da maioria dos itens voltados para o primeiro estágio do trabalho de parto. Já a taxa de adesão da prática do item 3 "Realização do toque vaginal pelo profissional a cada quatro horas" foi de $6(15 \%)$, recomendação que menos obteve adesão entre os profissionais. Em relação ao segundo estágio do trabalho de parto, os itens 2 e 3 foram os que alcançaram $22(55 \%)$ e $20(50 \%)$, respectivamente, sendo a menor adesão da categoria. No terceiro estágio, destaca-se a adesão máxima de 40 (100\%) correspondente ao item 1 "Uso de uterotônicos". Conclui-se que os profissionais obtiveram uma boa taxa de adesão em relação às práticas avaliadas no checklist, sempre buscando meios para reduzir os danos relacionados ao parto. Ademais, evidenciou que os partos assistidos por esses profissionais possuem menos intervenções e foram permeados pelas boas práticas. Palavras-chave: Segurança do Paciente; Cuidados de Enfermagem; Enfermagem Obstétrica; Parto Humanizado; Lista de Checagem.
\end{abstract}

\begin{abstract}
Given the context of the use of technologies to support good practices in childbirth, the aim of this study was to apply the intrapartum care checklist in vaginal birth. Descriptive, cross-sectional study with a quantitative approach, developed during the months of November and December 2020, in the delivery rooms of two hospitals, one secondary and the other tertiary. The study sample consisted of convenience, totaling 40 professionals. The instrument data were grouped, tabulated and transferred to the Excel program. Descriptive data analysis was performed using relative and absolute frequencies. It was observed that professionals followed the recommendations of most items aimed at the first stage of labor. The rate of adherence to the practice of item 3 "Performance of vaginal touch by the professional every four hours" was 6 (15\%), a recommendation that obtained less adherence among professionals. Regarding the second stage of labor, items 2 and 3 were the ones that reached $22(55 \%)$ and $20(50 \%)$, respectively, being the lowest adherence in the category. In the third stage, there is a maximum adherence of $40(100 \%)$ corresponding to item 1 "Use of uterotonics". It is concluded that the professionals obtained a good rate of adherence in relation to the practices evaluated in the checklist, always looking for ways to reduce the harm related to childbirth. Furthermore, it showed that births attended by these professionals have fewer interventions and were permeated by good practices.

Keywords: Patient Safety; Nursing Care; Obstetric Nursing; Humanizing Delivery; Checklist.

\footnotetext{
${ }^{1}$ Enfermeira. Residência em Enfermagem Obstétrica. Universidade Estadual do Ceará, Fortaleza, Brasil, https://orcid.org/0000-0002-4949-5898.

${ }^{2}$ Enfermeira. Universidade Estadual do Ceará, Fortaleza, Brasil, https://orcid.org/0000-0001-7594-0898.

${ }^{3}$ Enfermeira. Especialista em Enfermagem Obstétrica. Residência em Enfermagem Obstétrica. Mestre em Cuidados Clínicos em Enfermagem e Saúde. Professora do Curso de Graduação em Enfermagem. Universidade Estadual do Ceará, Fortaleza, Brasil, https://orcid.org/0000-0002-6203$\underline{0024}$

${ }^{4}$ Enfermeiro. Especialista em Enfermagem Obstétrica. Especialista em Enfermagem Clínica. Mestre em Saúde Coletiva. Doutor em Saúde Coletiva. Professor da Graduação em Enfermagem e da Pós-graduação em Saúde Coletiva. Universidade Estadual do Ceará, Fortaleza, Brasil. https://orcid.org/0000-0002-9483-8060.

${ }^{5}$ Enfermeira, Especialista em Unidade Terapia Intensiva. Doutora em Enfermagem Fundamental. Professora do Programa de Pós Graduação em Cuidados Clínicos e Saúde. Membro da Rede Brasileira de Segurança do Paciente e da Sociedade Brasileira de Segurança do Paciente. Universidade Estadual do Ceará, Fortaleza, Brasil, https://orcid.org/0000-0002-3406-9685
} 


\section{INTRODUÇÃO}

A gestação é marcada por diversas mudanças físicas e psicológicas, tornando o parto e o nascimento de uma criança eventos marcantes na vida da mulher e de sua família. Nesse contexto, durante esse estágio, ocorrem profundas alterações psicológicas, orgânicas e fisiológicas, repercutindo psíquica e socialmente na vida da mulher e de seus familiares ${ }^{(1)}$.

Diante disso, a assistência adequada e segura à mulher no momento do parto, é imprescindível para que ela vivencie a experiência da maternidade da melhor forma possível. Nesse contexto, para garantir a segurança e o bem-estar da mulher durante o momento do parto, é indispensável uma atenção segura e de qualidade, sendo assim, a equipe de saúde deve estar pronta para acolher a gestante, como também, seus familiares, estando bem preparada e fundamentada cientificamente para realização de procedimentos e de eventuais intercorrências ${ }^{(2)}$.

Segundo a Organização Mundial de Saúde, dados alarmantes demonstram a importância de promover uma assistência segura e qualificada durante o parto. Dos mais de 130 milhões de nascimentos que ocorrem todos os anos, cerca de 303.000 resultam na morte da mãe, 2,6 milhões em nados-mortos e 2,7 milhões na morte de recém-nascidos nos primeiros 28 dias de vida. A maior parte destas mortes ocorrem em contextos de baixos recursos, podendo a maioria delas ser evitada $^{(3)}$.

Define-se morte materna aquela que ocorre durante a gestação ou até 42 dias após seu término. Pode ser qualquer causa relacionada ou agravada pela gravidez, mas não por causas acidentais ou incidentais. Em torno de $92 \%$ das mortes maternas são por causas evitáveis e ocorrem, principalmente, por hipertensão, hemorragia ou infecções ${ }^{(4)}$.

Nesse contexto, considera-se que o índice elevado de casos relacionados à morte materna é um problema a ser enfrentado em todo o mundo. O Brasil está inserido no grupo de países latino-americanos que conseguiram atingir grandes avanços em relação a redução de mortes relacionadas à gravidez ou parto no período de 1990 a 2013. Neste mesmo período, o país reduziu sua taxa de mortalidade materna em $43 \%{ }^{(5)}$. Apesar de ter registrado importante redução da Razão de Mortalidade Materna (RMM) nos últimos anos, passando de 104 mortes maternas por 100.000 nascidos vivos, em 1990, para 44 em 2015 , não alcançou a meta do $5^{\circ}$ Objetivo de Desenvolvimento do Milênio ( $5^{\circ} \mathrm{ODM}$ ), que era reduzir em 75\% a RMM entre 1990 e $2015^{(6)}$.

Com o objetivo de modificar a realidade das altas taxas de mortalidade materna e neonatal, ressalta-se a importância da criação e, posteriormente, da aplicação de 
tecnologias para promover as boas práticas na assistência ao trabalho de parto. Percebe-se que, na evolução da assistência à saúde e na perspectiva do cuidado de enfermagem, as tecnologias vêm sendo planejadas e implementadas levando em consideração a necessidade de traduzir o conhecimento técnico-científico em ferramentas, processos, manuais e materiais criados ou utilizados para difundir tal conhecimento e, assim, melhorar a qualidade da assistência ${ }^{(7)}$.

Buscando oferecer uma assistência segura e baseada em evidências científicas, a Organização Mundial de Saúde (OMS) divulgou um Modelo de Cuidados intraparto para uma experiência positiva no parto. Esse manual contém recomendações na assistência ao processo de nascimento, parto e puerpério e, além disso, práticas não recomendadas, visando um cuidado menos intervencionista e mais respeitoso ${ }^{(8)}$. Sendo essa uma iniciativa internacional que necessita ser adaptada e aplicada na realidade brasileira.

Nessa perspectiva, adotar checklists e protocolos que promovam a segurança na assistência pode trazer benefícios para os profissionais e pacientes, além de envolver completamente a equipe de saúde, visto que melhora a comunicação entre os profissionais e reduz os eventos adversos na atenção ${ }^{(9)}$. Assim, observa-se a necessidade de desenvolver tecnologias que facilitem a promoção da segurança no parto, ressaltando os cuidados recomendados para essas mulheres e seus filhos nesse momento tão natural e particular.

Nesse sentido, o estudo justificase devido às altas taxas de mortalidade materno-infantil, ao longo dos anos, como também, de violência obstétrica com o uso de intervenções desnecessárias, que visam acelerar o processo fisiológico e natural do parto, bem como, comprometem diretamente a segurança da mãe e do bebê.

A verificação dos cuidados intraparto por meio de um checklist, baseado nas recomendações da OMS, beneficiará a população e a comunidade científica, principalmente a equipe multiprofissional que presta assistência às gestantes no momento do parto, visto que o estudo poderá estabelecer recomendações objetivas para um cuidado seguro a mulher e ao seu filho. Ademais, objetiva-se desmistificar práticas hospitalocêntricas que não são indicadas na assistência à mulher em trabalho de parto e resgatar o protagonismo feminino no processo de nascimento.

Diante do contexto, o presente estudo tem como objetivo aplicar o checklist de cuidados intraparto no parto vaginal.

\section{MÉTODOS}

Trata-se de um estudo descritivo, transversal de abordagem quantitativa, 
desenvolvido durante os meses de novembro e dezembro de 2020, nas salas de parto de dois hospitais, um de nível secundário e outro terciário, ambos foram escolhidos devido serem referências no atendimento obstétrico no estado do Ceará, como também, em relação ao quantitativo de partos vaginais. Os meses de coleta de dados foram reduzidos aos meses de novembro e dezembro devido à pandemia da COVID-19, período em que as instituições permitiram a entrada do pesquisador nas unidades.

Em ambas as instituições, o atendimento inicial das gestantes é realizado pelo enfermeiro, no acolhimento com classificação de risco. Em seguida, a paciente é encaminhada para o atendimento com o médico, que decide a necessidade ou não de internamento. Os partos normais são assistidos por médicos e enfermeiros obstetras, residentes de enfermagem e medicina em obstetrícia e internos de medicina.

No total, 80 profissionais atuam nas salas de parto das respectivas instituições, no entanto a amostra do estudo foi constituída por conveniência totalizando um quantitativo de 40 profissionais. Participaram da pesquisa os profissionais da saúde que estavam de plantão na sala de parto e que estavam assistindo ativamente a mulher durante o parto vaginal no período da coleta dos dados: médicos, enfermeiros, residentes e internos de enfermagem e medicina. Foram excluídos os profissionais que estavam de férias ou de licença durante o período da coleta de dados ou que iniciaram e não puderam concluir o parto.

A coleta de dados foi realizada pela pesquisadora, de segunda a quinta no horário de 07:00 às 19:00 horas. A coleta foi realizada da seguinte maneira: inicialmente a pesquisadora abordava o profissional que iria prestar assistência ao parto e convidava-o a participar da pesquisa. No momento da abordagem, foi realizado todos os esclarecimentos em relação ao presente estudo.

Em seguida, após o atendimento do parto, a pesquisadora enviava para o profissional, via whatsapp, o instrumento (checklist) de cuidados intraparto via Google Formulário, de modo a evitar o contato com materiais (papel, caneta, prancheta) que pudesse, de alguma forma, ser vetor de transmissão de infecções. O formulário online foi preenchido pelo profissional através do seu próprio aparelho celular. Ressalta-se que, apesar da pesquisadora permanecer no ambiente onde a assistência era prestada, em nenhum momento interferiu nas práticas dos profissionais.

A coleta de dados foi realizada por meio do Instrumento (checklist) de cuidados intraparto, validado no estudo de desenvolvimento de um checklist para 
cuidados intraparto, o qual passou pela submissão ao comitê de ética e validação do conteúdo com enfermeiros especialistas ${ }^{(10)}$. Esse instrumento foi baseado no documento lançado pela Organização Mundial da Saúde denominado Intrapartum care for a positive childbirth experience, que contém as recomendações para cuidados no trabalho de parto (TP) e parto ${ }^{(8)}$.

No checklist, os cuidados intraparto são divididos nas seguintes categorias: os cuidados para todo o processo de nascimento, os cuidados para o primeiro estágio do trabalho de parto, para o segundo estágio, para o terceiro estágio e por fim, os cuidados com o recém-nascido ${ }^{(8)}$. O instrumento de coleta avalia os cuidados, apenas, do processo de trabalho de parto, compreendido do primeiro ao terceiro estágio.

No primeiro estágio do trabalho de parto recomenda-se: usar as definições dos estágios de TP, no qual o primeiro estágio é: latente (colo até $5 \mathrm{~cm}$ ) e ativo (colo $>5 \mathrm{~cm}$ ). Realizar o controle intermitente dos batimentos cardiofetais (BCFs) com Sonar doppler ou Pinard, a cada 15 a 30 minutos. O toque vaginal a cada quatro horas. Promover o alívio da dor, tendo como opções a analgesia epidural ou opióides parenterais (fentanil, diamorfina e petidina) e as medidas não farmacológicas, como as técnicas de relaxamento, massagens e compressas. Permitir a ingestão de líquidos e alimentos, pelas gestantes com baixo de risco de necessitar de anestesia geral. Encorajar a movimentação, dando apoio à parturiente e estimulando a posição vertical, caso ela aceite $(8,11)$

Seguindo para o segundo estágio do trabalho de parto, deve-se considerar que a duração pode ser variável, sendo que geralmente é inferior a duas horas em multíparas e três horas em nulíparas. A posição deve ser de escolha da mulher, desde que o parto não apresente complicações. Orientar a paciente a realizar o puxo (empurrar) apenas seguindo o seu próprio impulso. Recomenda-se técnicas para reduzir o trauma perineal, como massagem perineal, compressa quente e proteção perineal com as mãos. Ademais, recomenda-se o controle intermitente dos BCFs com Sonar doppler ou Pinard a cada 5 minutos ${ }^{(8,11)}$.

Por fim, no terceiro estágio, recomenda-se a administração de ocitocina e se não estiver disponível, é recomendado o uso de outro uterotônico, como a ergometrina/metilergometrina ou misoprostol. Tração controlada do cordão, além disso, retardar o clampeamento do cordão, se não houver contraindicação, por pelo menos 1 minuto ${ }^{(8)}$.

Os dados do instrumento foram agrupados e tabulados no Google Formulário e em seguida, automaticamente foram transferidos para o programa Excel. A análise 
descritiva dos dados foi realizada por meio de frequências relativas e absolutas.

\section{RESULTADOS}

Participaram da pesquisa 40 profissionais. Destes, 18 (45\%) eram enfermeiros, 10 (25\%) residentes em enfermagem obstétrica, seis médicos residentes de obstetrícia e ginecologia (15\%) e seis $(15 \%)$ médicos obstetras.

Os profissionais da enfermagem assistiram 28 (70\%) partos. Sendo assim, cada profissional foi avaliado uma vez, resultando em um parto para cada profissional. Quanto a observação da adesão dos profisssionais às práticas recomendadas pela OMS para os cuidados no trabalho de parto (TP) e parto, os resultados foram divididos em três tabelas, cada uma representando o estágio do parto correspondente.

A tabela 1 corresponde às práticas recomendadas pela OMS no primeiro estágio do TP. Nota-se que a maior parte dos profissionais seguiram as recomendações da maioria dos itens.

Tabela 1 - Taxa de adesão das práticas recomendadas no primeiro estágio do trabalho de parto (N=40), Fortaleza, Ceará, Brasil, 2021.

\begin{tabular}{|c|c|c|c|c|}
\hline & $\begin{array}{r}\text { Ações } \\
\text { rea }\end{array}$ & $\begin{array}{l}\text { dáticas } \\
\text { das }\end{array}$ & $\begin{array}{l}\text { Ações e práticas } \\
\text { recomendadas }\end{array}$ & Adesão \\
\hline & $\begin{array}{l}\text { Sim } \\
\mathrm{n}(\%)\end{array}$ & $\begin{array}{l}\text { Não } \\
\mathrm{n}(\%)\end{array}$ & & $\mathrm{n}(\%)$ \\
\hline $\begin{array}{l}\text { 1) Usei as definições do estágio de TP: primeiro estágio } \\
\text { latente (colo até } 5 \mathrm{~cm} \text { ) e ativo (colo> } 5 \mathrm{~cm} \text { ). }\end{array}$ & $39(97,5)$ & $1(2,5)$ & Sim & 97,5 \\
\hline $\begin{array}{l}\text { 2) Realizei o controle intermitente dos batimentos } \\
\text { cardiofetais (BCF) com Sonar Doppler ou Pinard a cada } \\
15 \text { a } 30 \text { minutos. }\end{array}$ & $35(87,5)$ & $5(12,5)$ & Sim & 87,5 \\
\hline $\begin{array}{l}\text { 3) Realização do toque vaginal pelo profissional a } \\
\text { cada quatro horas }\end{array}$ & $6(15)$ & $34(85)$ & Sim & 15 \\
\hline
\end{tabular}

4) Utilizei métodos para alívio da dor (opióides parenterais; como fentanil, diamorfina e petidina) e as $\begin{array}{llll}39(97,5) & 1(2,5) & \text { Sim } & 97,5\end{array}$ medidas não farmacológicas, como as técnicas de relaxamento, massagem e compressas).

5) Permiti a ingestão de líquidos e alimentos pelas 
Nos itens 1 "Usei as definições do estágio de TP: primeiro estágio latente (colo até $5 \mathrm{~cm})$ e ativo (colo> $5 \mathrm{~cm})$ " e 4 "Utilizei métodos para alívio da dor (opióides parenterais; como fentanil, diamorfina e petidina) e as medidas não farmacológicas, como as técnicas de relaxamento, massagem e compressas)" a taxa de adesão a essas práticas foi de 39 (97,5\%), seguindo de 38 (95\%) do item 5 "Permiti a ingestão de líquidos e alimentos pelas gestantes que tem baixo risco de anestesia geral", e de $35(87,5 \%)$ dos itens 2 "Realizei o controle intermitente dos batimentos cardiofetais (BCF) com Sonar
Doppler ou Pinard a cada 15 a 30 minutos" e item 6 "Encorajei a movimentação da parturiente e o uso de posições verticais ou a posição que ela preferiu" (Tabela 1).

Ressalta-se que a adesão da prática do item 3 "Realização do toque vaginal pelo profissional a cada quatro horas" foi de seis $(15 \%)$ profissionais, recomendação que menos obteve adesão entre os profissionais (Tabela 1).

A tabela 2 corresponde às práticas recomendadas no segundo estágio do TP. Nota-se que as menores adesões dos profissionais foram em relação aos itens 2 e 3.

Tabela 2 - Taxa de adesão das práticas recomendadas no segundo estágio do trabalho de parto (N=40), Fortaleza, Ceará, Brasil, 2021.

\begin{tabular}{|c|c|c|c|c|}
\hline & \multicolumn{2}{|c|}{$\begin{array}{c}\text { Ações e práticas } \\
\text { realizadas }\end{array}$} & $\begin{array}{l}\text { Ações e práticas } \\
\text { recomendadas }\end{array}$ & $\begin{array}{l}\text { Adesão } \\
\mathbf{n}(\%)\end{array}$ \\
\hline & $\mathbf{n}(\%)$ & $\mathbf{n}(\%)$ & & \\
\hline 1) Considerar que a duração do segundo estágio pode ser & $40(100)$ & 0 & $\operatorname{Sim}$ & 100 \\
\hline 2) Orientei a parturiente a realizar o puxo (empurrar) apenas & $22(55)$ & $18(45)$ & Sim & 55 \\
\hline
\end{tabular}


3) Realizei técnicas para reduzir o trauma perineal, como massagem perineal, compressa quente e proteção perineal com as mãos.
$20(50) \quad 20(50) \quad 50$

4) Monitorei de forma intermitente os batimentos

cardiofetais (BCFs) com Sonar doppler ou Pinard a cada $5 \quad 37(92,5) \quad 3(7,5) \quad$ Sim $\quad 92,5$ minutos

Fonte: Elaborada pela autora

$\mathrm{O}$ item 1 "Considerar que a duração do segundo estágio pode ser variável, sendo que geralmente é inferior a duas horas em multíparas e três horas em nulíparas", destacou-se atingindo $40(100 \%)$ de adesão entre os profissionais, seguido do item 4 "Monitorei de forma intermitente os batimentos cardiofetais (BCFs) com Sonar doppler ou Pinard a cada 5 minutos", que obteve 37 (92,5\%) de adesão (Tabela 2).
Os itens 2 "Orientei a parturiente a realizar o puxo (empurrar) apenas seguindo seu próprio impulso" e 3 "Realizei técnicas para reduzir o trauma perineal, como massagem perineal, compressa quente e proteção perineal com as mãos" alcançaram $22(55 \%)$ e 20 (50\%) de adesão, respectivamente (Tabela 2).

A tabela 3 corresponde às práticas recomendadas no terceiro estágio do TP.

Tabela 3 - Taxa de adesão das práticas recomendadas no terceiro estágio do trabalho de parto $(\mathrm{N}=40)$, Fortaleza, Ceará, Brasil, 2021

\begin{tabular}{|c|c|c|c|c|}
\hline & Açõe & $\begin{array}{l}\text { Práticas } \\
\text { zadas }\end{array}$ & $\begin{array}{l}\text { Ações ou Práticas } \\
\text { Recomendadas }\end{array}$ & Adesão \\
\hline & $\begin{array}{l}\text { Sim } \\
\mathrm{n}(\%)\end{array}$ & $\begin{array}{l}\text { Não } \\
\text { n(\%) }\end{array}$ & & $\mathrm{n}(\%)$ \\
\hline $\begin{array}{l}\text { 1) Uso de uterotônicos (ocitocina, ergometrina/metilergometrina } \\
\text { ou misoprostol) para prevenir a hemorragia pós-parto (HPP). }\end{array}$ & $40(100)$ & 0 & Sim & 100 \\
\hline $\begin{array}{l}\text { 2) Realizar a tração controlada do cordão e retardar o } \\
\text { clampeamento do cordão, se não houver contraindicação, por pelo } \\
\text { menos } 1 \text { minuto. }\end{array}$ & $37(92,5)$ & $3(7,5)$ & Sim & 92,5 \\
\hline
\end{tabular}

Fonte: Elaborada pela autora

Destaca-se a adesão de todos os profissionais $40(100 \%)$ ao item 1 "Uso de uterotônicos (ocitocina, ergometrina/metilergometrina ou misoprostol) para prevenir a hemorragia pós-parto (HPP)", seguido do item 2 "Realizar a tração 
controlada do cordão e retardar o clampeamento do cordão, se não houver contraindicação, por pelo menos 1 minuto" que atingiu $37(92,5 \%)$ de adesão por parte dos profissionais (Tabela 3 ).

\section{DISCUSSÃO}

A enfermagem foi a categoria profissional predominante em relação à assistência e acompanhamento durante o TP. Nos últimos anos, a enfermagem obstétrica tem ganhado espaço nas políticas públicas de saúde devido ao seu olhar qualificado e humanizado do processo de parturição, aplicando esforços para que flua naturalmente sem necessidade de intervenções dispensáveis (12).

Nesse sentido, a enfermeira obstétrica tem como objetivo seguir um modelo de assistência que tenha o intuito de resgatar valores como o protagonismo, a individualidade, a privacidade, bem como, a autonomia da mulher, favorecendo um parto seguro e respeitoso.

O documento da OMS denominado Intrapartum care for a positive childbirth experience, no qual o instrumento foi baseado, contem recomendações para os cuidados durante o trabalho de parto e parto, e é direcionado principalmente aos profissionais da saúde responsáveis por desenvolvimento de protocolos de saúde nacionais e locais e aqueles que prestam cuidados diretamente a mulheres grávidas e seus recém-nascidos em todos os ambientes. Isso inclui parteiras, enfermeiras, clínicos gerais, obstetras e gestores de maternoinfantil dos programas de saúde ${ }^{(8)}$.

$$
\text { Em relação aos dados }
$$

apresentados sobre a adesão às práticas recomendadas do checklist, baseado no modelo de cuidados intraparto da OMS, os resultados apontam que a taxa de adesão das práticas recomendadas no primeiro estágio do trabalho referente ao item 1 "Usei as definições do estágio de TP: primeiro estágio latente (colo até $5 \mathrm{~cm})$ e ativo $($ colo $>5 \mathrm{~cm})$ " obteve $39(97,5 \%)$ de adesão profissional.

O primeiro estágio latente é um período de tempo caracterizado por contrações uterinas dolorosas e variáveis, alterações do colo do útero, incluindo algum grau de apagamento e progressão mais lenta da dilatação até $5 \mathrm{~cm}$. O primeiro estágio ativo é um período de tempo caracterizado por contrações uterinas dolorosas regulares, um grau substancial de apagamento cervical e dilatação cervical maior que $5 \mathrm{~cm}{ }^{(8)}$.

Ressalta-se que durante a fase latente é essencial a realização da assistência de enfermagem individualizada, realização de orientações e técnicas de respiração e relaxamento. Nesse contexto, é importante orientar a parturiente a ficar em posição vertical (de pé, caminhando ou sentada) ou 
em decúbito lateral, estas posições possibilitam maior intensidade e maior eficiência das contrações ${ }^{(13)}$.

A prática recomendada no item 2

"Realizei o controle intermitente dos batimentos cardiofetais (BCF) com Sonar Doppler ou Pinard a cada 15 a 30 minutos" atingiu 35 (87,5\%) de adesão. Quanto a essa prática, é fundamental durante a assistência de enfermagem no trabalho de parto a avaliação contínua tanto do feto quanto da parturiente, a avaliação da dinâmica uterina, monitoramento dos batimentos cardiofetais, sinais vitais, monitoramento deperda de líquido amniótico, sangue, diminuição dos batimentos fetais e sinais de desconfortos apresentados pela parturiente $^{(14)}$

A prática recomendada no item 3 "Realização do toque vaginal pelo profissional a cada quatro horas" foi de 6 (15\%), destaca-se que essa recomendação obteve a menor adesão entre os profissionais. Diante desse resultado, justifica-se que a rotina das instituições hospitalares observadas ainda preconiza o toque vaginal a cada duas horas. O exame de toque vaginal em intervalos de quatro horas é recomendado para avaliação de rotina da primeira fase do trabalho de parto em mulheres de baixo risco (8).

Em relação a prática recomendada no item 4 "Utilizei métodos para alívio da dor (opioides parenterais; como fentanil, diamorfina e petidina) e as medidas não farmacológicas, como as técnicas de relaxamento, massagem e compressas)" a taxa de adesão a essas práticas foi de 39 (97,5\%).

Segundo dados da WHO, a maioria das mulheres deseja alguma forma de alívio farmacológico ou não farmacológico da dor durante o trabalho de parto, e evidências qualitativas indicam que a massagem pode reduzir o desconforto durante o trabalho de parto, aliviar a dor e melhorar a experiência materna ${ }^{(8)}$.

Dados de uma pesquisa descritiva, qualitativa, sobre a eficiência de métodos não farmacológicos para alívio da dor no trabalho de parto normal, realizada com 40 puérperas na maternidade do Hospital e Maternidade Sagrado Coração de Jesus em Janaúba-MG, evidenciou que os métodos mais utilizados foram banho de aspersão, deambulação, mudanças de posições (cócoras, sentada, agachamento), técnicas de controle da respiração, massagens e bola suíça e obtiveram efeitos satisfatórios, pois minimizaram a sensação dolorosa e deixaram as gestantes mais tranquilas e relaxadas ${ }^{(15)}$.

$\mathrm{O}$ uso de tecnologias nãofarmacológicas para alívio da dor identificados na pesquisa $(97,5 \%)$ apresentou percentual bem acima dos $(19,1 \%)$ na região Nordeste, identificados na pesquisa sobre o Nascer no Brasil, um estudo nacional de base hospitalar composto por puérperas e seus 
recém-nascidos (16). Enquanto que se assemelha ao estudo transversal sobre práticas na assistência ao parto em maternidades com inserção de enfermeiras obstétricas, em Belo Horizonte, Minas Gerais que apontou $(74,2 \%)$ de uso desses métodos. Esses índices podem ser considerados um aspecto positivo das práticas realizadas na instituição, mostrando um cuidado humanizado que promove o bemestar da parturiente ${ }^{(17)}$.

De acordo com a prática recomendada no item 5 "Permiti a ingestão de líquidos e alimentos pelas gestantes que tem baixo risco de anestesia geral", a adesão foi de 35 (87,5\%). Estudos apontam que não existem evidências científicas que apoiem a restrição de líquidos e alimentos durante o trabalho de parto para as parturientes com baixo risco de complicações. Nesse sentido, dados de pesquisa desenvolvida na cidade do Recife sobre fatores associados à violência obstétrica na assistência ao parto vaginal em uma maternidade de alta complexidade em Recife, Pernambuco apontam que $80 \%$ das mulheres ingeriram líquidos durante $\mathrm{o}$ trabalho de parto ${ }^{(18)}$. Ademais, tal percentual foi semelhante ao presente estudo $(87,5 \%)$ e também ao encontrado no estudo quantitativo transversal retrospectivo, descritivo documental sobre o perfil da assistência ao parto em uma maternidade pública para o Centro Obstétrico Superior e Centro de Parto Normal $(74,8 \% \text { e } 98,7 \%)^{(19)}$.
No que diz respeito a prática recomendada no item 6 "Encorajei a movimentação da parturiente e o uso de posições verticais ou a posição que ela preferiu", que resultou em $35(87,5 \%)$ de adesão. No presente estudo, (87,5\%) encorajaram a movimentação da mulher, contrapondo-se aos $(44,3 \%)$ do estudo sobre intervenções obstétricas durante o trabalho de parto e parto em mulheres brasileiras de risco habitual ${ }^{(16)}$.

No que se refere as taxas de adesão das práticas recomendadas no segundo estágio do trabalho, os resultados apontam que houve adesão de todos os profissionais ao item 1 "Considerar que a duração do segundo estágio pode ser variável, sendo que geralmente é inferior a duas horas em multíparas e três horas em nulíparas”. O segundo estágio do TP é o período de tempo entre a dilatação cervical completa e o nascimento do bebê, durante o qual a mulher tem uma necessidade involuntária de realizar puxos/empurrar, em resposta à contração uterina $^{(8)}$.

A descrição do início do segundo período com base na pesquisa é uma ciência inexata e o início da segunda fase do parto na prática clínica muitas vezes não é conhecido com precisão. Nesse sentido, por exemplo, uma mulher pode sentir o desejo de realizar puxos antes da dilatação completa ou ela pode 
ainda não sentir esse desejo no momento em que dilatação completa é diagnosticada ${ }^{(8)}$.

Quando a condição da mulher é satisfatória, e o feto está em boas condições, e há evidências de progresso na descida da cabeça fetal, não há motivos para intervenção. No entanto, quando o segundo estágio se estende além das durações padrão acima mencionadas, a chance de parto espontâneo em um tempo razoável diminui, e a intervenção para acelerar o parto deve ser considerada $^{(8)}$.

Em relação ao item 2 "Orientei a parturiente a realizar o puxo (empurrar) apenas seguindo seu próprio impulso" foi alcançado 22 (55\%) de adesão profissional. Destaca-se que as mulheres na fase expulsiva do segundo estágio do parto devem ser encorajadas e apoiadas a seguirem seu próprio desejo de empurrar. Evidências qualitativas sobre o que é importante para as mulheres durante os cuidados no parto, mostram que deixar as parturientes seguirem seus instintos e vontades, proporciona autonomia e controle no processo do parto e nascimento ${ }^{(20)}$.

Os profissionais da saúde devem evitar impor empurrões dirigidos às mulheres na segunda fase do trabalho de parto, pois não há evidências de qualquer benefício com esta técnica ${ }^{(8,21)}$.

O estudo transversal e quantitativo desenvolvido em um hospital público de Setúbal em Portugal sobre desfechos perineais e as variáveis associadas no parto na água e no parto fora da água obteve uma taxa de $32,2 \%$ de partos fora da água que não foram realizados puxos dirigidos pelos profissionais, contrapondo-se ao presente estudo em que (55\%) dos profissionais não realizaram os puxos dirigidos, prática recomendada pela OMS ${ }^{(22)}$.

De acordo com o item 3 "Realizei técnicas para reduzir o trauma perineal, como massagem perineal, compressa quente e proteção perineal com as mãos", obteve-se 20 (50\%) de adesão por parte dos profissionais. Para mulheres na segunda fase do parto, técnicas para reduzir o trauma perineal $\mathrm{e}$ facilitar parto espontâneo (incluindo massagem perineal, compressas mornas e proteção "com as mãos" do períneo) são recomendados, com base nas preferências da mulher e nas opções disponíveis ${ }^{(8)}$.

As evidências sugerem que a massagem perineal pode aumentar a chance de manter o períneo intacto e reduz o risco de lesões perineais graves, que as compressas perineais quentes reduzem lesões perineais de terceiro e quarto grau, e que a proteção perineal com as mãos, provavelmente reduz as lesões perineais de primeiro grau ${ }^{(8)}$.

Ressalta-se que a técnica handson, na qual contêm o períneo durante o segundo período do trabalho de parto espontâneo de gestações únicas, resulta em incidência semelhante de vários traumas 
perineais em comparação com a técnica hands-off, na qual o assistente não realiza nenhum tipo de proteção perineal durante o segundo período do trabalho de parto. A incidência de lacerações de terceiro grau e episiotomia aumenta com a técnica hands-on (23).

Nesse contexto, não há suficiente evidência de que o hands-on de fato previna qualquer tipo de lesão obstétrica. É possível que o uso dessa técnica ajude na prevenção de lesões para alguns casos específicos de trabalho de parto, mas, por outro lado, é possível que para outros casos, como nos partos de multíparas, a técnica seja inclusive prejudicial. Contudo estes são apontamentos débeis do ponto de vista da evidência científica, e novos estudos com melhor qualidade metodológica se fazem urgentes para responder definitivamente estas intrigantes questões ${ }^{(24)}$.

No que se refere ao item 4 "Monitorei de forma intermitente os batimentos cardiofetais (BCFs) com Sonar doppler ou Pinard a cada 5 minutos", obtevese $37(92,5 \%)$ de adesão. Há evidências que sugerem que a ausculta intermitente (AI) com um Sonar Doppler Fetal, cardiotocografia (CTG) ou monitoramento rigoroso com estetoscópio fetal de Pinard pode aumentar a detecção de anormalidades da frequência cardíaca fetal (FCF), o que pode, por sua vez, reduzir os desfechos de hipóxia neonatal ${ }^{(8)}$.
O protocolo de ausculta consiste em um intervalo de ausculta a cada 15-30 minutos no primeiro estágio ativo do trabalho de parto e a cada 5 minutos na segunda fase do trabalho de parto. Além disso, a duração da ausculta deve ser de pelo menos 1 minuto, se o FCF nem sempre estiver no faixa normal (ou seja, 110-160 bpm), a ausculta deve ser prolongada durante pelo menos três contrações. Deve-se realizar a ausculta durante uma contração uterina e continuar por pelo menos 30 segundos após a contração. Além disso, deve-se realizar o registro da FCF basal (como um único número contado em batimentos por minuto) e a presença ou ausência de acelerações e desacelerações. Ressalta-se que independentemente do método utilizado, uma explicação clara da técnica e sua finalidade deve ser fornecido à mulher ${ }^{(8)}$.

No que se refere a taxa de adesão das práticas recomendadas no terceiro estágio do trabalho de parto, os resultados apontam adesão de todos os profissionais ao item 1 "Uso de uterotônicos (ocitocina, ergometrina/metilergometrina ou misoprostol) para prevenir a hemorragia pós-parto (HPP)". $\mathrm{O}$ uso de uterotônicos para a prevenção da hemorragia pós-parto (HPP) durante a terceira fase do trabalho de parto é recomendado para todos os partos. A ocitocina (10 UI, IM / IV) é o medicamento uterotônico recomendado 
para a prevenção do pós-parto hemorragia (HPP) ${ }^{(8)}$.

Um estudo transversal, descritivo e analítico, com 222 mulheres, sobre o uso de uterotônicos no terceiro período do parto em uma maternidade da Zona da Mata Mineira, evidenciou que em (80\%) dos partos vaginais foi utilizado uretônico intramuscular, assemelhando-se ao encontrado no presente estudo 40 (100) ${ }^{(25)}$.

Em relação ao item 2 "Realizar a tração controlada do cordão e retardar o clampeamento do cordão, se não houver contraindicação, por pelo menos 1 minuto" atingiu 37 (92,5\%) de adesão por parte dos profissionais. Estudos apontam que bebê que foi submetido ao clampeamento tardio teve menor chance de necessitar de algum tipo de suporte ventilatório ou transfusão sanguínea. Além disso, seu estoque de ferro e de hemoglobina foram, na maioria das vezes, superiores àquele que não realizou essa prática. Ademais, demonstrou efeitos positivos ao RN, o que sugere que essa estratégia seja realizada em toda assistência neonatal ${ }^{(19)}$.

Diante do exposto, os profissionais que prestaram assistência aos partos e responderam o checklist durante o período de coleta de dados, demonstraram uma boa adesão a maioria das práticas recomendadas no instrumento.
Ressalta-se ainda, que as práticas que tiveram baixa adesão podem ser apresentadas aos diretores e profissionais da instituição e trabalhadas através de palestras, treinamentos, capacitações e implementação de protocolos e cheklists.

Quanto as limitações do estudo, destaca-se a dificuldade em relação à adesão dos profissionais à pesquisa, visto que se trata de um estudo que avalia as práticas assistenciais prestadas e muitos apresentaram uma certa resistência logo no início da abordagem, principalmente os profissionais médicos. Ainda, devido a pandemia pelo COVID-19 surgiram algumas restrições a restrição de coleta de dados nas instituições hospitalares e o atraso no processo de aprovação da pesquisa pelos comitês de ética, devido ao funcionamento e trabalhadores reduzidos.

\section{CONCLUSÕES}

Diante dos resultados, pode-se concluir que o objetivo de identificar a adesão dos profissionais às práticas avaliadas pelo checklist, recomendadas pela Organização Mundial da Saúde, foi atingido com êxito, e que as taxas de adesão às práticas foram satisfatórias na maioria dos itens.

Conclui-se que os profissionais obtiveram uma boa taxa de adesão em relação às práticas, sempre buscando meios para 
reduzir os danos relacionados ao parto, garantindo a segurança materna e neonatal.

$\mathrm{O}$ presente estudo evidenciou que os partos assistidos pela enfermagem obstétrica, possuem menos intervenções e são permeados pelas boas práticas, destacando a importância da atuação deste profissional no trabalho de parto e parto vaginal de risco habitual.

Diante disso, é possível afirmar que a enfermagem obstétrica vem contribuindo com a humanização no parto e nascimento, garantindo cada vez mais uma assistência segura e qualificada para a mulher e seu bebê.

A aplicação do checklist para cuidados intraparto mostrou-se simples, direta e de fácil entendimento e preenchimento por parte dos profissionais. Além disso, proporcionou a listagem das recomendações baseadas em evidências durante o trabalho de parto, demonstrando que os profissionais podem fornecer uma assistência menos intervencionista, mais respeitosa e dentro dos critérios éticos da autonomia, mantendo ou melhorando os resultados perinatais.

A utilização do checklist destacou-se por apresentar as recomendações a serem seguidas pelos profissionais, como também, por proporcionar às mulheres que receberam os cuidados seguros e de qualidade, um momento único e particular.
Por fim, espera-se que o checklist possa ser aplicado de forma rotineira na prática assistencial obstétrica, promovendo melhorias e que possa gerar outros novos estudos na área.

Nesse contexto, um país como o Brasil, onde encontramos barreiras em diversas áreas, sendo algumas socioeconômicas, culturais, e políticas, uma prática simples e de baixo custo, como a implementação do checklist de cuidados intraparto, beneficiando uma assistência segura e de qualidade deve ser incentivada e apoiada.

\section{REFERÊNCIAS}

1. Alves TV, Bezerra MMM. Principais alterações fisiológicas e psicológicas durante o Período Gestacional. Id on Line Rev. Mult. Psic. 2020; 14 (49) 114-26.

2. Pereira SS, Oliveira ICMS, Santos JBS et al. Parto natural: a atuação do enfermeiro diante da assistência humanizada. Tempus Actas de saúde coletiva. 2016;10 (3); 199213.

3. Organização Mundial Da Saúde. Lista de Verificação da OMS para Partos Seguros [Internet]. Geneva: 2018 [citado em 2021 jun. 03]. Disponível em: http://www.who.int/patientsafety/implementat ion/checklists/childbirth_portuguese/en/.

4. Ministério da Saúde (BR). Agência Saúde. Ministério da Saúde investe na redução da mortalidade materna [Internet]. Brasília: 2018 [citado em 2021 jun. 03]. Disponível em:https://www.saude.gov.br/noticias/agencia -saude/43325-ministerio-da-saude-investe-nareducao-da-mortalidade-materna. 
5. Organização Das Nações Unidas. Mortes Maternas [Internet]. Geneva: OMS; 2017 [citado 2021 jun. 03] Disponível em: https://Nacoesunidas.Org/?Post_type=Post $\& S$ =Mortes+Maternas. Acesso Em: 05 Jan 2020.

6. Dantas SLC, Oliveira GYM, Costa KFL et al. Estudos experimentais no período gestacional: panorama da produção científica. Revista Escola Enferm USP. 2018,52.

7. Barbosa EMG, Sousa AAS, Vasconcelos FGM et al. Tecnologias educativas para promoção do (auto) cuidado de mulheres no pós-parto. Rev Bras Enferm,2016, 69(3),54553.

8. World Health Organization. Recommendations: intrapartum care for a positive childbirth experience [Internet]. Geneva: 2018 [citado em 2021 jun 07]. Disponível em:https://apps.who.int/iris/bitstream/handle/ 10665/260178/9789241550215eng.pdf? sequence $=1$.

9. Boeckmann LMM, Rodrigues MCS. Adaptação e validação de checklist de segurança cirúrgica na cesárea. Texto Contexto Enferm, 2018, 27(3).

10. Rebouças BDS. Desenvolvimento de um checklist para cuidados intraparto [Trabalho de Conclusão de Curso]. Fortaleza: Curso de Enfermagem, Universidade Estadual do Ceará; 2021.70 p.

11. Federação Brasileira das Associações de Ginecologia e Obstetrícia (FEBRASGO). Reunião de Mortalidade Materna FEBRASGO/OPAS [Internet]. São Paulo: 2018 [citado em 2021 jun 07]. Disponível em: https://www.febrasgo.org.br/pt/noticias/item/ 685-reuniao-de-mortalidade-maternafebrasgo-opas.

12. Duarte MR, Alves VH, Rodrigues DP et al Tecnologias do cuidado na enfermagem obstétrica: contribuição para o parto e nascimento. Cogitare enferm [Internet], 2019[citado em 2021 jun 07], 24:e54164.Disponível http://www.revenf.bvs.br/pdf/ce/v24/1414-8536-ce24-e54164.pdf.

13. Lima MFG, Pequeno AMC, Rodrigues DP et al. Desenvolvendo competências no ensino em enfermagem obstétrica: aproximações entre teoria e prática. Rev Bras Enferm. 2017;70(5):1110-6.

14. Nascimento ACA, Lima ALP, Araújo JC et al. Assistência de enfermagem na fase latente do trabalho de parto: Relato de experiência. International nursing congress. 2017;1(1):9-12.

15. Dias EG, Ferreira ARM, Martins AMC et al. A eficiência de métodos não farmacológicos para alívio da dor no trabalho de parto normal. Enferm. Foco, 2018; 9(2): 35-39.

16. Leal MC, Pereira APE, Domingues RMSM et al. Intervenções obstétricas durante o trabalho de parto e parto em mulheres brasileiras de risco habitual. Cad Saúde Pública, 2014,30:17-32.

17. Sousa AMM, Souza KV, Rezende EM et al. Práticas na assistência ao parto em maternidades com inserção de enfermeiras obstétricas, em Belo Horizonte, Minas Gerais. Esc Anna Nery [online], 2016 [citado em 07 jun de 2021], 20(2). Disponível em: https://doi.org/10.5935/1414-8145.20160044.

18. Andrade PON, Silva JPQ, Diniz CMM, Caminha MFC. Fatores associados à violência obstétrica na assistência ao parto vaginal em uma maternidade de alta complexidade em Recife, Pernambuco. Rev Bras Saúde Mater Infant. 2016, 16(1),29-37.

19. Resende MTS, Lopes DS, Bonfim EG. Perfil da assistência ao parto em uma maternidade pública. Rev. Bras. Saúde Mater. Infant., Recife, jul-set. 2020, 20 (3): 871-878.

20. Downe S, Finlayson K, Oladapo OT, Bonet M, Gülmezoglu AM. What matters to women duringchildbirth: a systematic qualitative review? PLoS One. 2018;13(4):e0194906. doi: 10.1371/journal.pone.0194906. eCollection 2018.

21. Ministério da Saúde (BR). Secretaria de Ciência, Tecnologia e Insumos Estratégicos. Departamento de Gestão e Incorporação de 
Tecnologias em Saúde. Diretrizes nacionais de assistência ao parto normal [Internet]. Brasília, 2017 [citado em 2021 jun 07]. Disponível em: http://bvsms.saude.gov.br/bvs/publicacoes/diretrize s_nacionais_assistencia_parto_normal.pdf.

22. Camargo JCS, Varela V, Ferreira FM et al. Desfechos perineais e as variáveis associadas no parto na água e no parto fora da água: estudo transversal. Rev. Bras. Saúde Mater. Infant. Recife, out-dez 2019; 19 (4):787-796.

23. Williams RAMP, Saccone G, Berghella V. Hands-on versus hands-off techniques for the prevention of perineal trauma during vaginal delivery: a systematic review and meta-analysis of randomized controlled trials. The Journal of Maternal-Fetal \& Neonatal Medicine.2019;34 (6).

24. Lima EN, Bueno KJOB, Nunes EFC, Latorre GFS. Hands-on durante o período expulsivo: herói ou vilão? Rev Pesqui Fisioter. 2020;10(2):346-354. doi: 10.17267/2238-2704rpf.v10i2.2810

25. Ayres LFA, Santos KEA, Beirigo BA, Lima VD, Prado MRMC, Henriques BD, Passos CM. Uso de uterotônicos no terceiro período do parto em uma maternidade da Zona da Mata Mineira. REME - Rev Min Enferm. 2020[citado em 2021 jun 07];24:e-1344. Disponível em: http://reme.org.br/artigo/detalhes/1520. DOI: 10.5935/1415.2762.20200081

\section{Autor correspondente}

Luana Sousa de Carvalho, Rua Capitão Américo Menezes, 455, Parangaba, 60710130, Fortaleza-CE.

(85)987961502, luanasousa741@gmail.com.

Fomento e Agradecimento: Universidade Estadual do Ceará.

Submissão: 2021-09-17

Aprovado: 2021-09-26 